\title{
Acceptability Evaluation of Inter-driver Interaction via a Vehicle Agent Using Vehicle-to-Vehicle Communication on a Driving Simulator
}

\author{
ToshiYuki HagiYa ${ }^{1, \text { a) }} \quad$ KAZUNARI NAWA $^{1, b)}$ \\ Received: January 19, 2021, Accepted: July 7, 2021
}

\begin{abstract}
Vehicle-to-vehicle ( $\mathrm{V} 2 \mathrm{~V})$ communication is expected to serve as a new information exchange method to help reduce traffic accidents. Most current services can notify drivers of dangers predicted by exchanging vehicle information. However, human drivers use multiple modalities to express their intentions, such as gestures and lights as well as vehicle behavior, even if these interactions can cause misunderstandings. A solution to reduce these misunderstandings is to exchange driver intentions via V2V communication. This study proposes the concept of exchanging driver intentions via a driver agent that understands the driver's utterance and sends a message using $\mathrm{V} 2 \mathrm{~V}$ communication. An experiment including five scenarios was conducted by employing the Wizard of $\mathrm{Oz}$ method on a driving simulator. The acceptability of the inter-driver interaction was evaluated by conducting a questionnaire survey and a semi-structured interview. Furthermore, we analyzed the relationship between acceptability and one's usual driving style. Consequently, we formed hypothesized key factors of the inter-driver interaction via V2V communication.
\end{abstract}

Keywords: Vehicle-to-vehicle (V2V) communication, acceptability evaluation, inter-driver interaction

\section{Introduction}

Numerous people have been losing their lives due to traffic accidents. Even in Japan, where transportation systems and infrastructure have been well-developed, over 400,000 accidents occur annually [1]. Traffic accidents have various causes, including insufficient safety confirmation and misunderstanding of interactions. Therefore, numerous driving support systems have been studied to help reduce traffic accidents. Among these systems, driving support using vehicle-to-vehicle (V2V) communication is expected to be employed as a new information exchange method to help reduce traffic accidents [2].

Currently, most existing and potential V2V communication services involve notifying drivers of an increased risk of collision with another vehicle when changing position or speed (e.g., intersection movement assist [3], [4], [5], [6]). However, in actual traffic environments, drivers often use multiple modalities (e.g., gestures, headlight flashing, and horns) to warn other drivers while negotiating a potentially risky maneuver [7], [8]. For example, impatient drivers may activate their headlights to force the front vehicle to move to another lane. In these situations, information exchange is limited; thus, such interactions can lead to misunderstandings or conflicts. Additionally, some conventional interactions (like a line of sight and gestures) are not effective for autonomous vehicles. Recently, interaction methods between autonomous vehicles and pedestrians have attracted increased at-

\footnotetext{
Toyota Motor Corporation, Chiyoda, Tokyo 100-0004, Japan

toshiyuki_hagiya@mail.toyota.co.jp

kazunari_nawa_aa@mail.toyota.co.jp
}

tention. Similarly, the interaction between autonomous vehicles and human-driven vehicles remains a problem that requires attention [9], [10], [11], [12]. A potential solution to improve interactions between human-driven vehicles is to exchange driver intentions via V2V communication. An example is a scenario wherein a driver requests to overtake the front vehicle by applying $\mathrm{V} 2 \mathrm{~V}$ communication rather than flashing their headlights. Although some studies have proposed use cases and a communication protocol for inter-driver interaction, the acceptability of drivers has not yet been investigated. Accordingly, we have simulated a V2Vbased inter-driver interaction and evaluated its acceptability [13].

This study proposes a concept in which a vehicle agent understands the intention of a driver's utterance and sends a message to another vehicle. The acceptability of the interaction is evaluated using the Wizard of $\mathrm{Oz}$ (WoZ) method. We conduct an experiment that comprises five scenarios: right turn at an intersection, lane change, overtaking, merging on a highway, and parking. The acceptability is evaluated by conducting questionnaire surveys and semi-structured interviews. Acceptability is influenced by a driver's personality and driving style; thus, we also analyze the relationship between acceptability and one's usual driving style obtained from a driving style check sheet [14]. This study has three main contributions: 1) it clarifies the acceptability of interdriver interaction through a vehicle agent using V2V communication; 2) it demonstrates the differences in acceptability for the two roles of sending and receiving requests; and 3) it analyzes the relationship between acceptability and driving style. 


\section{Related Work}

\subsection{Inter-driver Interaction}

Renner et al. compared face-to-face interaction with interdriver interaction by considering basic research on inter-driver interaction [8]. Their results showed that inter-driver interaction depends on the driver's gestures, vehicle signal systems, and preset traffic rules, while face-to-face interaction greatly depends on verbal interaction. Researchers recently proposed new methods for expressing the driver's intention by employing various modalities, including light and sound [9], [10], [11], [12]. Dey et al. proposed a concept for expressing intentions like body language by substantially changing the light position and color [15]. However, using a physical interaction method limits both information quality and quantity. A study demonstrated that an ambiguous interaction can lead to an aggressive driving behavior [16]. Therefore, numerous studies using $\mathrm{V} 2 \mathrm{~V}$ communication have been conducted to reduce traffic misunderstandings.

\subsection{V2V Communication}

$\mathrm{V} 2 \mathrm{~V}$ communication is a technology that allows talking among nearby vehicles [6]. A study by the National Highway Traffic Safety Administration (NHTSA) showed that V2V communication can reduce up to $80 \%$ of crashes [2]. Over the last decade, many researchers have proposed protocols and concepts to alert drivers of upcoming potential hazards by exchanging information with other vehicles using V2V communication [3], [4], [5], [6]. Based on information on the motion of neighboring vehicles, Sengupta et al. presented the concept of a cooperative collision warning system that provides warnings and situation awareness displays to drivers [3]. The study stated that V2V communication can improve safety and maintain a comfortable inter-vehicle distance. Makanae et al. proposed a prototype that utilizes a wireless local area network and a GPS to send messages to vehicles around a vehicle [17]. Verroios et al. proposed a method for limiting the range of communication by utilizing a motion sensor and a GPS [4]. Moreover, techniques have been proposed to identify which vehicle communicates with one's vehicle by combining the vehicle position from $\mathrm{V} 2 \mathrm{~V}$ with the relative position and features from sensors such as an image sensor and a radar [18]. For example, a cooperative adaptive cruise control system determines and establishes communication with the front vehicle by combining positions from $\mathrm{V} 2 \mathrm{~V}$ with radar information [19]. This is one of the promising techniques to let drivers identify the interaction vehicle.

\subsection{Social Message Interaction}

Not only exchanging information directly related to accidents but also the transmission of social messages have recently been studied. Wang et al. presented "CarNote," which is a concept of reducing misunderstanding and conflicts between drivers by displaying their emergency driving status to others. Their results showed that CarNote enhances drivers' empathy, increases forgiveness, and decreases anger toward others on the road [20]. For a real-time interaction with other vehicles, Wang et al. developed the concept of detecting gestures and sending them to nearby vehicles [21]. Lamas et al. also conducted an interview survey on the necessary requirements for sending messages through intervehicle communication [12] and obtained three requirements: 1) understanding the sender of the message, 2) interface with few distractions, and 3) easy to send a predetermined message. The voice interface is a strong candidate. As aforementioned, interactions using inter-vehicle interaction have been widely studied. However, to the best of our knowledge, no experimental investigation has yet been conducted on the acceptability of inter-driver interaction.

\section{Selection of Traffic Scenarios}

This section describes the selection of scenarios used in the experiments. From the use cases released by some associations such as the 5G Automotive Association [22] and NHTSA [23], we first considered approximately 20 use cases of $\mathrm{V} 2 \mathrm{~V}$ communication that required negotiation. These use cases include passing on a narrow road, overtaking across the centerline, turning at an intersection, merging onto a highway, overtaking on a highway, and entering a priority road from a non-priority road, etc.

Based on the use cases, we developed two hypotheses related to the acceptability of inter-driver interaction. One is that use cases to solve current traffic problems would be highly acceptable. The other is that acceptability is strongly influenced by several factors with regard to the relationship with other vehicles. Next, based on the hypotheses, we extracted five factors related to acceptability as shown in Table 1. Two factors in terms of current traffic problems are a high number of accidents and tailgating. The other three in terms of the relationship with other vehicles are the location and direction of the vehicle initiating communication, the time constraints for interaction, and an alternative interaction method from the perspective of both the sender and the receiver.

Finally, five scenarios (i.e., right turn, lane change, overtaking, merging, and parking) were selected so that the factors would not overlap in each scenario. The selected use cases and the factor hypothesis related to acceptability are shown in Table 1 . The right

Table 1 Five use cases and hypotheses factors involved in the acceptability of driver communication.

\begin{tabular}{|c|c|c|c|c|c|}
\hline \multirow[b]{2}{*}{ Hypotheses factors } & \multicolumn{5}{|c|}{ Use cases } \\
\hline & Right turn & Lane change & Overtaking & Merging & Parking \\
\hline Traffic problems & Most common accidents & $\mathrm{N} / \mathrm{A}$ & Tailgating & N/A & N/A \\
\hline $\begin{array}{l}\text { Location and direction of vehicle } \\
\text { to initiate communication }\end{array}$ & Opposite lane & $\begin{array}{l}\text { Different lane in the } \\
\text { same direction }\end{array}$ & Same lane & $\begin{array}{l}\text { Different lane in the } \\
\text { same direction }\end{array}$ & Parking \\
\hline Time constraints & Applied & $\mathrm{N} / \mathrm{A}$ & N/A & Applied & $\mathrm{N} / \mathrm{A}$ \\
\hline Alternative means for the sender & Turn signal & Turn signal & Passing & Turn signal & No \\
\hline Alternative means for the receiver & Turn signal & $\begin{array}{l}\text { No (when } \\
\text { running parallel) }\end{array}$ & $\begin{array}{l}\text { Depends on } \\
\text { the case }\end{array}$ & $\begin{array}{l}\text { No (when } \\
\text { running parallel) }\end{array}$ & No \\
\hline
\end{tabular}


turn scenario was adopted because it involves the highest number of accidents ${ }^{* 1}$. The overtaking scenario was adopted as a typical example of tailgating. The remaining three were adopted because factors did not overlap and communication would be considered effective.

\section{Experiment}

We employed a driving simulator to simulate the interaction with other vehicles using $\mathrm{V} 2 \mathrm{~V}$ communication as shown in Fig. 1). In this simulation, a vehicle agent mediates the driver's interaction via $\mathrm{V} 2 \mathrm{~V}$ communication. The vehicle agent understands the initial request through the driver's utterance and sends the request to another vehicle using $\mathrm{V} 2 \mathrm{~V}$ communication. In the following subsections, we describe the scenarios, the experiment structure, the participants, and the experimental conditions.

\subsection{Scenarios}

Five scenarios (i.e., right turn, lane change, overtaking, merging, and parking) were implemented in the simulator. In all scenarios, the participants played both roles of sending and receiving requests. A description of each scenario is provided below.

Right turn scenario: Fig. 2 (a) presents the right turn scenario.

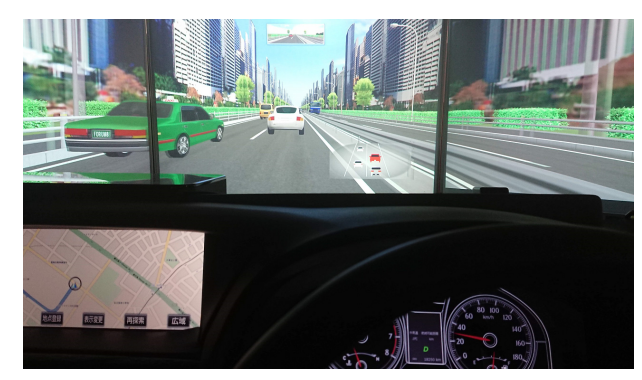

Fig. 1 Driving simulator screen.

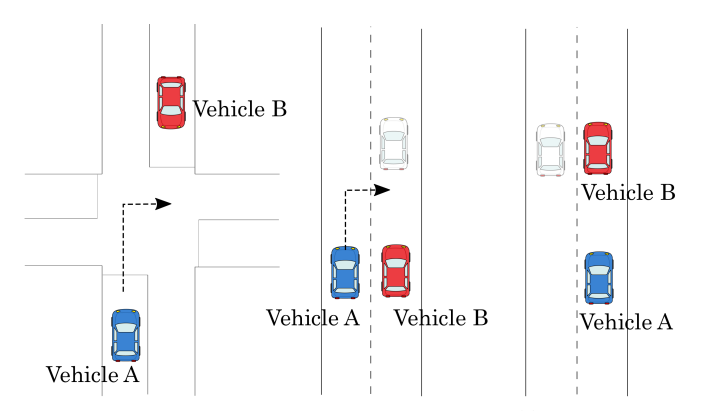

(a) Right turn

(b) Lane change

(c) Overtaking
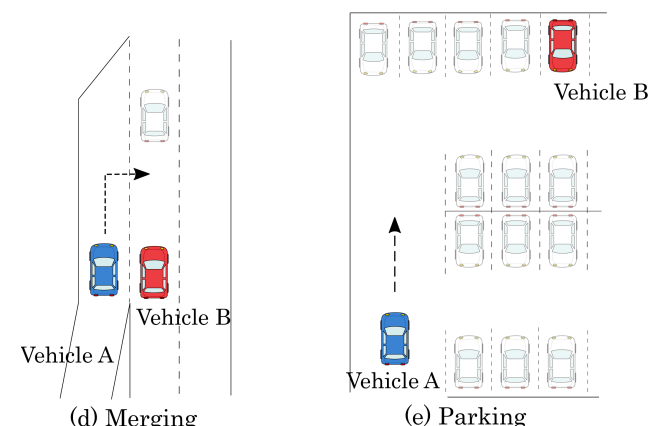

(e) Parking

Fig. 2 Inter-vehicle interaction scenarios: Vehicle A sends a request, and Vehicle $\mathrm{B}$ receives a request.

*1 https://www.itarda.or.jp/

Vehicle A is traveling at $40 \mathrm{~km} / \mathrm{h}$ for $100 \mathrm{~m}$ before an intersection with no traffic lights. First, the driver of Vehicle A instructs his/her vehicle agent to convey a request that he/she wishes to turn right to vehicle $\mathrm{B}$, located in the opposite lane. The agent of Vehicle $B$ then receives the message and sends it to the driver of Vehicle B by a synthesized voice and a head-up display (HUD). In response, the driver of Vehicle $\mathrm{B}$ approves the request by voice and stops the vehicle. The driver of Vehicle A is informed of the approval and turns right.

Lane change scenario: Fig. 2 (b) presents the lane change scenario. Vehicles A and B are traveling on the left and right sides of a two-lane road, respectively, at $80 \mathrm{~km} / \mathrm{h}$. The driver of Vehicle A instructs the vehicle agent to convey a request that he/she wants to change lanes to that of Vehicle B. The agent of Vehicle B then receives the message and sends it to the driver. In response, the driver of Vehicle B approves the request and accelerates the vehicle. The driver of Vehicle $\mathrm{A}$ is informed of the approval and changes lanes.

Overtaking scenario: Fig. 2 (c) presents the overtaking scenario. Vehicles A and B are traveling on the right side of a twolane road at $80 \mathrm{~km} / \mathrm{h}$. First, the driver of Vehicle A instructs the vehicle agent to convey a request that he/she wants to overtake Vehicle B. The agent of Vehicle B then receives the message and sends it to the driver. In response, the driver of Vehicle B approves the request and accelerates the vehicle, and changes lanes to yield. The driver of Vehicle A is informed of the approval and accelerates to overtake Vehicle B.

Merging scenario: Fig. 2 (d) presents the merging scenario. Vehicles $A$ is traveling in the merge lane at $60 \mathrm{~km} / \mathrm{h}$, attempting to merge into the main lane of a highway. First, the driver of Vehicle $\mathrm{A}$ instructs the vehicle agent to convey a request that he/she wants to merge to Vehicle B, which is traveling at $80 \mathrm{~km} / \mathrm{h}$ in the main lane. The agent of Vehicle B then receives the message and sends it to the driver. In response, the driver of Vehicle B approves the request and slows down to yield. The driver of Vehicle $\mathrm{A}$ is informed of the approval and accelerates to merge.

Parking scenario: Fig. 2 (e) presents the parking scenario, in which Vehicle A is searching for a parking space in a parking lot. First, the driver of Vehicle A instructs the agent to search for a parking space. The agent broadcasts the request to surrounding vehicles. The agent of Vehicle $B$ receives the message and sends it to the driver. In response, the driver of Vehicle B responds that he/she will depart immediately. The driver of Vehicle A is informed of the reply and heads in the direction of Vehicle B.

\subsection{Experiment Structure}

To reduce the burden on the participants, the experiments were divided into two parts. The use cases were separated to avoid overlapping factors related to acceptability listed in Table 1. Right turn and merging scenarios were divided into different parts because the two had a common characteristic in that there is a time constraint. Lane changing and merging scenarios were also divided into different parts because the two were similar in the location and direction of the vehicle initiating communication. As a result, Experiment 1 included the right turn, lane change, and overtaking scenarios, and Experiment 2 included the merging and 


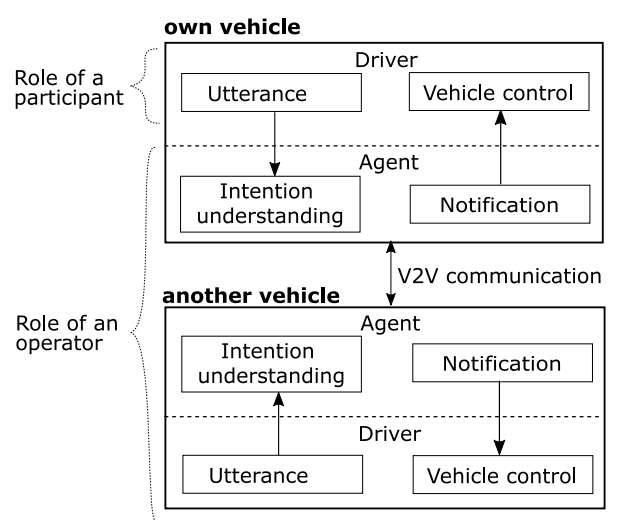

Fig. 3 Roles of participants and operators.

parking scenarios.

Experiments 1 and 2 were conducted at an interval of at least three months so that prior knowledge of the experiment would not affect the results. Initially, we planned to recruit the same participants in both experiments; however, due to recruiting problems, different participants were included in Experiments 1 and 2. Whether the participants' difference or similarity in Experiments 1 and 2 influenced the results, was statistically verified in Section 5 .

\subsection{Participants}

In Experiment 1, 23 participants took part in the experiment (i.e., 21 males and 2 females, aged 24 to 55 years with a mean age of $40.0(\mathrm{sd}=10.0))$. In Experiment 2, 21 participants took part in the experiment (i.e., 18 males and 3 females, aged 24 to 55 years with a mean age of $39.1(\mathrm{sd}=9.6))$. Ten of the participants were the same participants as in Experiment 1 (i.e., 8 males and 2 females, aged 24 to 55 years with a mean age of $42.4(\mathrm{sd}=10.2)$. All participants had driver licenses, and none had used voice interfaces before. In addition, none had medical problems, including problems affecting the visual and auditory senses.

The project of this investigation was approved and conducted according to the "Ethical Guidelines for Research" of Toyota Motor Corporation. Informed consent was obtained from all participants including details of the experimental procedures and our privacy protection policies.

\subsection{Apparatus}

This experiment was conducted utilizing the WoZ method, wherein the behavior of the vehicle agent and other vehicles was operated by a human operator. Figure 3 presents the roles of the participants and the operator. The roles of the participants were to control a vehicle on a driving simulator and make or accept a request by voice. Participants could operate the steering wheel, the accelerator, the brakes, and the turn signal, and they played the roles of the driver of Vehicle $\mathrm{A}$ in the sending request scenario and the driver of Vehicle $\mathrm{B}$ in the receiving request scenario. The operator controlled the timing of the request or acceptance and the action of the vehicle after acceptance by the key operation assigned to the keyboard. A synthesized voice was played when the key operation for the request and acceptance was performed. The operator performed the key operation approximately three sec-

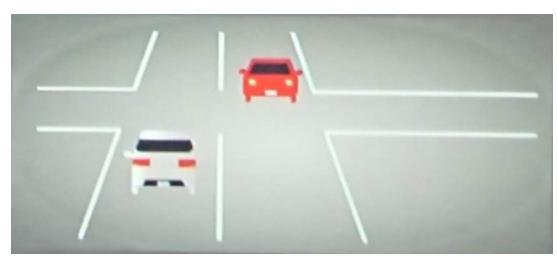

Fig. 4 Head-up display notification.

onds after the participant's request, referred to the reaction time of participants in the preliminary experiment. Figure 4 illustrates that the HUD displayed the relative positions of neighboring vehicles. The driving simulator screen comprised three projector screens, and the HUD and rearview mirror were presented on the screen. The driving simulator used the FORUM8 UC-win/Road ver. 10 , and the software for speech synthesis was Open JTalk ${ }^{* 2}$.

\subsection{Procedure}

First, the participants completed a questionnaire that investigated their attributes. There were 19 questions, including age, gender, driving frequency, and 16 questions on driving style from a driving style questionnaire [14]. Next, the participants practiced driving a square course with a side length of $500 \mathrm{~m}$ in the clockwise direction for two laps to familiarize themselves with the driving simulator. The experiment was explained to the participants during practice (e.g., how to make a request and what was displayed on the HUD).

Next, we conducted the experiment by utilizing the driving simulator. For evaluation, participants compared with their usual driving without conducting a baseline experiment. The reason is that it is difficult to control the conditions because the baseline can be different for each participant for some use cases. For example, in an overtaking scenario, some participants may continue to drive behind without doing anything, while others may close the distance until the driver in front notices. The explanation of the scenario was displayed on the screen before each trial. We eliminated the effect of order by randomly selecting the order of the trials from combinations of the scenarios and two roles of sending and receiving requests. Each trial was repeated twice. In other words, there were 12 trials in Experiment 1 and eight trials in Experiment 2. Trials that the operator judged to lead to accidents were deemed unsuccessful, and the participants retried immediately after the trial.

After the experiment, the participants completed a 4-point Likert-scale questionnaire to investigate the acceptability of the interaction compared to their usual driving, in which 4 indicated a strong agreement. The questionnaire involved evaluating the following five items for each role scenario: Useful, Contribute to safe driving, Not unpleasant, Reliable, and Preferred. No matter whether the trials were successful or retried, participants answered questions in the same way. After completing the questionnaire, the participants underwent an interview.

\section{Experimental Results}

\subsection{Acceptability of Scenario}

Figure 5 presents the results of the questionnaire of Experi-

*2 http://open-jtalk.sourceforge.net 
ment 1 , that is, three scenarios of a right turn, lane change, and overtaking. The neutral score was 2.5 , as the questions had four levels. Sending requests had higher average scores than receiving requests. However, no statistically significant differences were found among the scenarios for any of the questions using the Kruskal-Wallis test for each question $(\alpha=0.05)$.

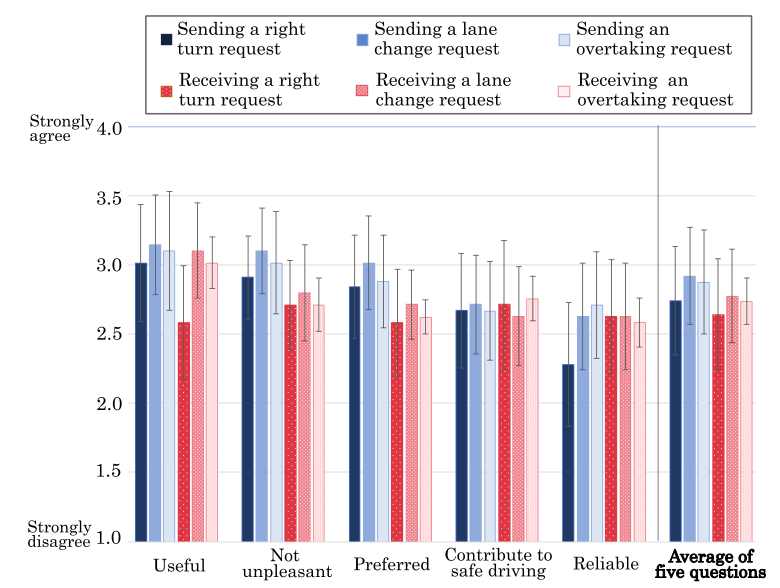

Fig. 5 Questionnaire average of Experiment 1; vertical bars show standard errors.

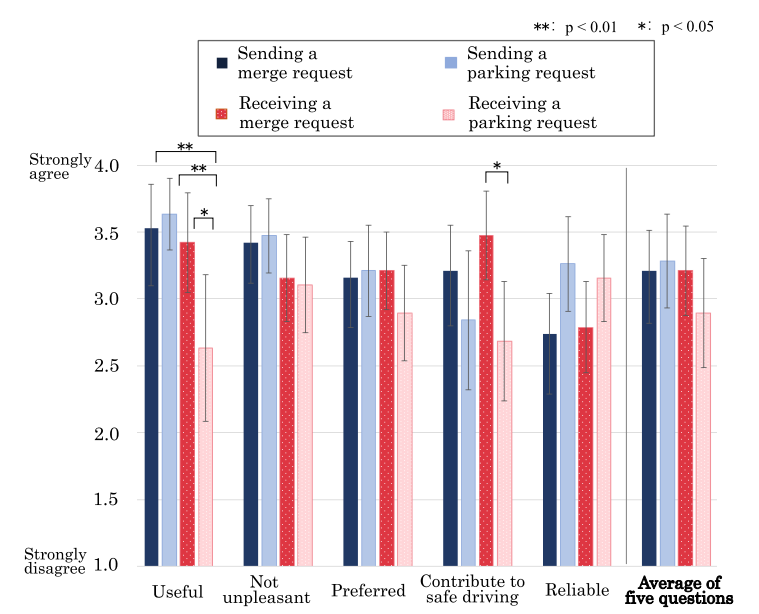

Fig. 6 Questionnaire average of Experiment 2; vertical bars show standard errors.
Figure 6 presents the results of the questionnaire of Experiment 2 , that is, the merging and parking scenarios. The graph was formed by mixing the two groups (i.e., the participants who joined and did not join in Experiment 1), as there was no statistical difference between the two groups using the Mann-Whitney $\mathrm{U}$ test. In terms of the average score, sending and receiving were almost the same in the merging scenario; however, the average score of receiving was lower in the parking scenario. Nevertheless, no statistically significant differences were found among the scenarios in terms of the average score using the Kruskal-Wallis test. For each question, the scenario of receiving a parking request had significantly lower scores than the other three scenarios for the Useful attribute, and significantly lower scores than the scenario of receiving a merging request for the Contributes to safe driving attribute by applying a Kruskal-Wallis test with the post hoc Steel-Dwass test $(\alpha=0.05)$.

Figure 7 presents the results of the questionnaire for participants who took part in both Experiments 1 and 2. The average scores and their order were generally similar to the values for all participants in Experiments 1 and 2. Sending requests had higher average scores than receiving requests. For each question, the scenarios of receiving a right turn request and a parking request had significantly lower scores than some scenarios for the Useful attribute by applying a Kruskal-Wallis test with a post hoc Steel-Dwass test ( $\alpha=0.05$ ). Similarly, the scenario of receiving a right turn request led to significantly lower scores than parking scenarios for Reliable.

\subsection{Relationship between Driving Style and Acceptability}

The analysis results of the relationship between driving styles and acceptability are described below. The following eight types of driving styles were calculated with a four level score from the scores of the driving style questionnaire [14]: Confidence in driving skills, Hesitation for driving, Impatience in driving, Methodical driving, Preparatory maneuvers at traffic signals, Importance of the automobile for self-expression, and Moodiness in driving.

Spearman's correlation coefficient and the p-value between the

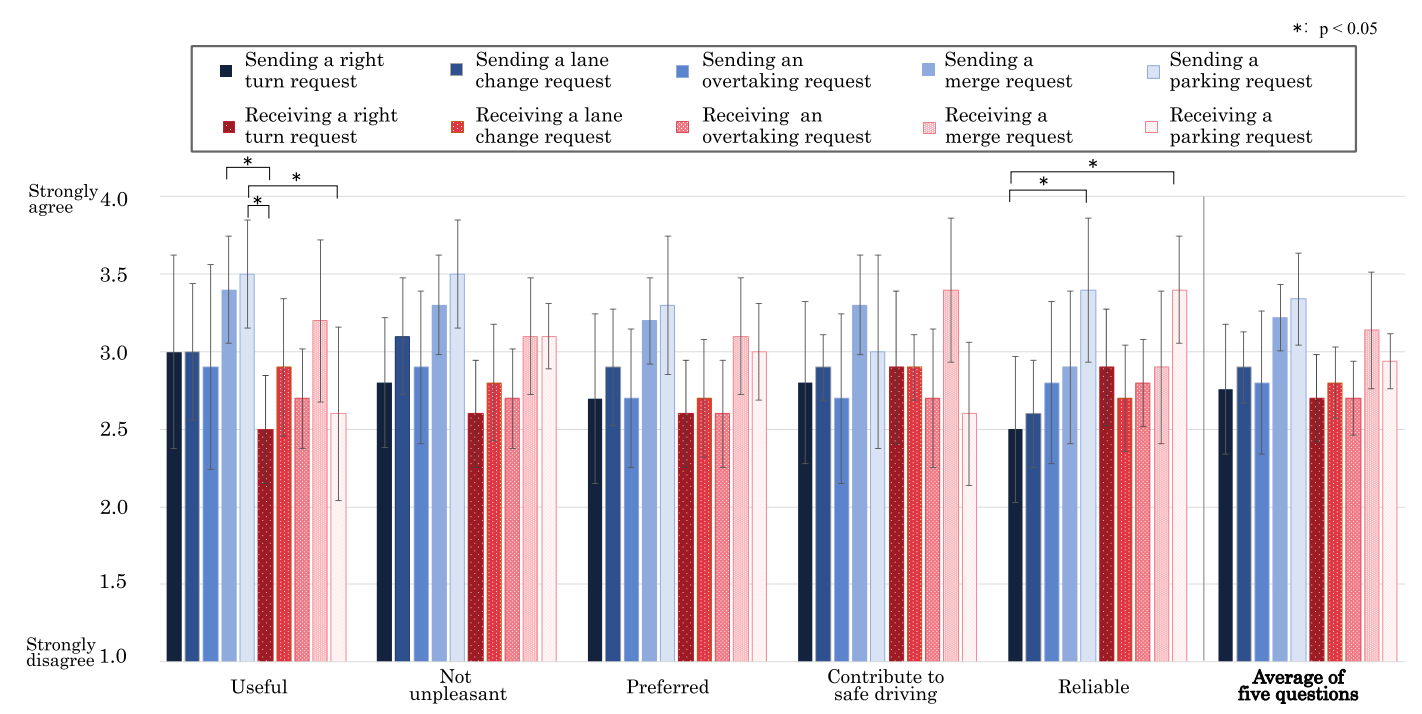

Fig. 7 Questionnaire average for participants who took part in both Experiments 1 and 2; vertical bars show standard errors. 
Table 2 Combinations of driving style and acceptability of inter-driver interaction with a statistically significant difference in Experiment 1.

\begin{tabular}{|c|c|c|c|}
\hline Driving style & Scenario & Question items & Correlation coefficient \\
\hline \multirow{2}{*}{$\begin{array}{l}\text { Confidence in } \\
\text { driving skills }\end{array}$} & Receiving request - Overtaking & Useful & $-0.55 *$ \\
\hline & Receiving request - Overtaking & Not unpleasant & $-0.40 *$ \\
\hline \multirow{5}{*}{$\begin{array}{l}\text { Hesitation for } \\
\text { driving }\end{array}$} & Sending request - Overtaking & Not unpleasant & $0.44 *$ \\
\hline & Sending request - Right turn & Contribute to safe driving & $0.44 *$ \\
\hline & Receiving request - Right turn & Reliable & $0.41 *$ \\
\hline & Receiving request - Overtaking & Useful & $0.41 *$ \\
\hline & Receiving request - Overtaking & Reliable & $0.41 *$ \\
\hline \multirow{3}{*}{$\begin{array}{l}\text { Impatience in } \\
\text { driving }\end{array}$} & Sending request - Right turn & Preferred & $-0.44 *$ \\
\hline & Sending request - Right turn & Contribute to safe driving & $-0.43 *$ \\
\hline & Sending request - Right turn & Reliable & $-0.56 * *$ \\
\hline \multirow{3}{*}{$\begin{array}{l}\text { Methodical } \\
\text { driving }\end{array}$} & Sending request - Right turn & Useful & $0.48 *$ \\
\hline & Sending request - Right Turn & Contribute to safe driving & $0.41 *$ \\
\hline & Receiving request - Right Turn & Useful & $0.58 * *$ \\
\hline
\end{tabular}

Table 3 Combinations of driving style and acceptability of inter-driver interaction with a statistically significant difference in Experiment 2.

\begin{tabular}{|c|c|c|c|}
\hline Driving style & Scenario & Question items & Correlation coefficient \\
\hline \multirow{3}{*}{$\begin{array}{l}\text { Impatience in } \\
\text { driving }\end{array}$} & Sending request - Merging & Useful & $-0.6 * *$ \\
\hline & Sending request - Merging & Contribute to safe driving & $0.55 *$ \\
\hline & Receiving request - Merging & Contribute to safe driving & $-0.64 * *$ \\
\hline \multirow{2}{*}{$\begin{array}{l}\text { Methodical } \\
\text { driving }\end{array}$} & Receiving request - Merging & Useful & $0.53 *$ \\
\hline & Receiving request - Merging & Contribute to safe driving & $0.46 *$ \\
\hline \multirow{2}{*}{$\begin{array}{l}\text { Anxiety about } \\
\text { traffic accidents }\end{array}$} & Sending request - Merging & Useful & $-0.47 *$ \\
\hline & Receiving reques - Merging & Not unpleasant & $-0.66 * *$ \\
\hline
\end{tabular}

driving style and each question were calculated for each scenario. Tables $\mathbf{2}$ and $\mathbf{3}$ present the combination of significant statistical correlations with a significance level of 0.05 in Experiments 1 and 2, respectively. The results demonstrate that the following five driving styles were statistically correlated with some questions for the scenarios: Confidence in driving skills, Hesitation for driving, Impatience in driving, Methodical driving, and Anxiety about traffic accidents. A statistically significant negative correlation was found between Confidence in driving skills and the scenario of receiving an overtaking request. A higher value of Hesitation for driving indicates a higher degree of unwillingness to drive and a stronger tendency to drive on well-equipped roads when driving is unavoidable. A statistically significant positive correlation was found between this driving style and the scenarios of sending/receiving an overtaking request and receiving a right turn request. For Impatience in driving, there was a positive correlation with the scenario of sending a merge request, and a negative correlation with the scenarios of sending a right turn request and receiving a merge request. Methodical driving had a statistically significant positive correlation with the items related to sending/receiving a right turn request and receiving a merge request. Anxiety about traffic accidents had a statistically significant negative correlation with items related to sending/receiving a merge request. The other three driving styles (Preparatory maneuvers at traffic signals, Importance of the automobile for selfexpression, and Moodiness in driving) had no statistically significant correlations with the questions for the scenarios. In addition, no statistically significant differences in the questions on lane change and parking were found based on driving styles.

\subsection{Qualitative Research}

Semi-structured interviews were conducted after the driving simulator experiment to obtain further feedback about the system. The questions pertained to the following topics: the overall concept, impressions of each scenario, and human-machine interface (HMI).

\subsubsection{Overall Concept}

The concept of inter-driver interaction via a vehicle agent was generally approved. Most participants appreciated the benefits of making each side aware of the other's intention, such as in the statement "I felt that the system improved safety by helping the other person become attentive when he/she was not aware" and from an ethical perspective, such as in the statement "I thought it was good to give way to others." However, one of the participants who had high confidence in the driving skills commented, "If this service becomes popular, I will turn it off if I get too many requests."

\subsubsection{Impressions of Each Scenario}

Most participants commented based on three aspects: comparison with usual driving, relation to traffic rules, and trust in the other driver.

In the right turn scenario, positive opinions were obtained, such as "It becomes possible to safely make a right turn by informing each other." However, numerous negative opinions were also expressed. Five participants provided the following comments based on the perspective of traffic rules: "Since priority is always given to those who go straight, I do not know why we need to give priority to those who turn right." In addition, four participants expressed the following opinion from the perspective of inconveniencing the other vehicle: "I felt uneasy asking the other vehicle to stop for me to turn right." Additionally, four participants who answered "Strongly disagree" for the Reliable attribute when sending a right turn request reported the following based on the perspective of trusting the other driver: "I felt uneasy because I 
did not know whether the other driver was going to immediately respond or not, and actually going to let me pass although he/she replied so."

The following positive opinions were obtained in the lane change scenario: "I felt that the system was effective because it is always difficult to change lanes when the surrounding traffic is congested" and "It is impossible to know whether a driver who drives parallel to me is going to change lanes or not, and it was good to know the intention from the request so that I could let the driver pass." Furthermore, one comment implied that the vehicle agent needed to possess more detailed information: "Sometimes I did not know whether I had to accelerate or decelerate to let the other driver get into my lane, so it is good to know exactly where the other driver wants to enter the lane."

In the overtaking scenario, opinions based on comparison with usual driving were as follows: "I feel uncomfortable when someone uses a headlight flashing from behind, so it is easier for me if the vehicle agent informs me that another vehicle is going to overtake" and "So far, there has been no means to inform a driver who slowly drives in the overtaking lane. This system is a good way to do that." The following opinion was also obtained: "Unlike the turn right scenario, there was enough time to inform the other driver, making this function easy to use." Negative opinions were obtained from the perspective of anxiety, such as "I felt anxious when I got the request because I felt rushed" and "I felt anxious to request because it could lead to trouble."

For the merging scenario, several positive comments were provided, such as "It is good to know the timing of the merging vehicle because I sometimes do not know if it is actually merging on a real highway." As suggestions for improvement, there were various comments, such as "When merging, it is difficult to know which vehicle I sent a request to" and "It would be nice to be guided to enter either in front of or behind the other vehicle," as same in the lane change scenario.

For the parking scenario, positive comments included, "It's good that it tells me when there is an available space," and "This feature lets me know if there is a vehicle that is trying to park, so I feel like I should get out of there as soon as possible." There were also suggestions for improvement, such as, "It would be better if the system told me when I entered the parking lot without asking," and "I would be glad if the agent automatically told vehicles around me when I was leaving."

\subsubsection{HMI}

Regarding receiving notification from the vehicle, most participants considered that combining voice notification and the screen display was effective. One opinion was: "It was easy to understand because I received the notification by both display and voice." Some participants also expressed positive opinions regarding the information to be displayed: "It was easy to know which vehicle responded by the vehicle icon flashing on and off in the HUD." However, one participant said, "The positional relationship with other vehicles was only displayed immediately after the request was received, so I did not know how much the vehicle agent was aware of the surrounding conditions."

\section{Discussion}

In this section, we discuss the following three research questions: the acceptability of the inter-driver interaction via a vehicle agent using V2V communication, the difference in the acceptability for the two roles of sending and receiving requests, and the relationship between acceptability and driving style.

\subsection{Acceptability of Inter-driver Interaction}

The score of the Useful attribute was the highest for all questions. Except for the scenarios of receiving right turn and parking requests, the score was 3.0 or higher, indicating that the system was approved because 2.5 is a neutral score compared to their usual driving. However, the score of the Reliable attribute was the lowest for all questions. On the other hand, in the comparison among the scenarios, the average score of merging and parking was the highest, followed by lane change, overtaking, and right turn. The scores of sending requests were generally higher than those of receiving requests. Based on these observations, we propose a hypothesis about the four factors related to the acceptability of the inter-driver interaction via a vehicle agent.

The first point is the presence or absence of alternative means. When turning right, a driver can use the turn signal without any inconveniences or hesitation. However, most drivers are reluctant to use headlight flashing when overtaking another vehicle. Similarly, the other vehicle cannot be informed of the intention to change a lane or merging onto the main line by a turn signal when both vehicles are traveling in parallel. In addition, there is no general way to know which vehicle is about to leave a parking lot. From these perspectives, this system can be effectively used in situations in which there is currently no means to convey one's intentions, and for interaction during unfamiliar situations (e.g., when responding to autonomous driving).

The second point is the relationship with traffic rules and manners. As a matter of course, drivers change lanes or merge onto a highway without hesitation. However, from the perspective of traffic manners, drivers are generally reluctant to use headlight flashing when overtaking another vehicle. Similarly, there is hesitation in requesting to turn right based on the perspective of traffic rules because incoming vehicles going straight are prioritized. When compared with usual driving, this difference in hesitation can be one of the reasons why differences in the Preferred and the Not unpleasant attributes were observed in each scenario.

The third point is sufficient interaction time. Parking does not involve time constraints in communication. In addition, when changing lanes, overtaking, and merging, the other vehicle is traveling in the same direction; therefore, there is sufficient time for interaction. In contrast, the interaction when turning right is with an oncoming vehicle, for which there is not sufficient time. Requesting to turn right can be directly linked to an accident if an interaction error occurs or the other driver acts differently from the expected response. Since the communication delay by the DSRC (dedicated short-range communications), one of the communication systems, is less than $10 \mathrm{msec}$ [24], almost all of the time is occupied for interaction. Therefore, it can show the same tendency in a real environment. This appears to be the reason why 
the Reliability attribute was very low when sending a right turn request. Based on this perspective, if a vehicle can autonomously judge and act through autonomous driving, Reliability can be improved because there will be no room for the other driver's decision, allowing sufficient time margin and improving consistency between the response and action.

The fourth point is the transparency of functions. Information on the surrounding vehicles was conveyed only during communication. Some participants commented, "I did not know to what extent the agent understood the surrounding traffic conditions." In recent years, transparency has been considered crucial in increasing the use of artificial intelligence. The same principle applies to this system. As an improvement, continually displaying the vehicle position can assure the driver that the vehicle knows its surroundings in real time. Furthermore, the decisions to make a request and give consent were made by the driver in this study. However, as sensing functions evolve and improve, it will be possible in the future to enable vehicles to make decisions and actions. Thus, an HMI to inform the driver to what extent the vehicle can understand its surroundings and perform actions is increasingly important.

\subsection{Difference in Acceptability of Roles}

Figures 5, 6, and 7 demonstrate that in sending requests, three items (Useful, Not uncomfortable, and Preferable) were higher than in receiving requests. One reason for this may be that some participants had a negative impression of communicating their intentions by the agent's notification. Drivers generally have a negative impression of forced ways of conveying intention (e.g., the headlight flashing and horns). The same impression appeared to apply to the agent's notification. The second potential reason, as observed in the parking scenario, is that some participants did not want to provide an additional response, as they commented that they wanted the agent to respond automatically. Another possible reason for this was that the HMI was not sophisticated. In the interview, some participants commented that they could not judge how to act when receiving a request, especially in the lane change scenario, although it was clear how to act when sending a request. Therefore, these two points indicate that improving acceptability may be achieved by improving the HMI.

\subsection{Relationship between Acceptability and Driving Style}

A significant correlation was observed between the items in the questionnaire and the following five items: Confidence in driving skills, Hesitation for driving, Impatience in driving, Methodical driving, and Anxiety about traffic accidents. The former two can be attributed to the driver's usual driving customs, while the latter three can be attributed to the driver's personality. Moreover, five out of seven items related to the scenario of receiving right turn and overtaking requests were correlated with the former two items. This implies that the more unfamiliar the driver is with driving, the more difficult it is for him/her to grasp the surrounding situation, and thus, the more receptive he/she is to receive requests. In contrast, the lane change, merging, and parking scenarios had no correlation with the driver's driving style. This implies that there are scenarios that are not custom-dependent. Ac- ceptability is dependent on the four hypotheses described earlier. For the personality aspects of the latter three items, drivers with cautious and meticulous personalities appeared to express higher acceptability in the scenarios. In contrast, drivers who were impatient and had confident personalities demonstrated less acceptability. Thus, some use cases, in which acceptability is greatly affected by a driver's personality should have a function that allows the driver to select whether to use it or not.

\section{Limitations and Future Work}

We conducted an experiment involving five scenarios using a driving simulator. However, an actual road environment has two major differences. The first point is that the impression and effectiveness of the proposed interaction are somewhat arbitrary owing to the limited situations of the scenarios. Although there should have been a scenario with heavy traffic, the scenarios simulated only low-traffic roads. One participant commented: "I think it feels different if the traffic differs even in the same turn right scenario. Although I did not agree with the situation of the experiment, I was willing to stop to give way for another vehicle turning right if it was crowded in front of the intersection." Additional experiments for simulating various environments should be conducted to identify better use cases of inter-vehicle interaction.

The second point is the difference in danger awareness of drivers between a driving simulator and an actual roadway. In the interview, three participants made the following similar comment: "I did not feel any danger in the driving simulator even though it displayed some dangerous behavior; thus, I cannot judge if this system would contribute to safety." Although simulating a dangerous environment is difficult, conducting experiments to simulate a dangerous environment is indispensable to perform a detailed subjective evaluation. The time interval from receiving a request to making a decision should be compared with various safety measures; for example, according to a survey conducted by the Ministry of Land, Infrastructure, Transport and Tourism, it takes $3.7 \mathrm{~s}$ to provide information, $3.2 \mathrm{~s}$ for alerting, and $0.8 \mathrm{~s}$ for warning [25].

\section{Conclusion}

In this study, we proposed a concept wherein a vehicle agent understands the intention of a driver's utterance and sends a message using V2V communication. We then evaluated the acceptability of the inter-driver interaction on a driving simulator utilizing the WoZ method. We conducted experiments for the following five scenarios: right turn, lane change, overtaking, merging, and parking. The acceptability was evaluated by questionnaire surveys and semi-structured interviews. We also analyzed the relationship between acceptability and a driver's usual driving style obtained from the driving style check sheet. The concept of inter-driver interaction via a vehicle agent was generally approved from the perspective of effectiveness. In some scenarios, driving customs and personality affected acceptability. In this study, we formed the hypothesis that four factors were related to acceptability, namely the presence or absence of alternative means, the driver's relationship with traffic rules and manners, sufficient interaction time, and transparency of functions. Based on this 
experiment, we hope to explore more effective usage scenarios in the future and evaluate the acceptability of interaction when agents and HMI become more sophisticated.

\section{References}

[1] National Police Agency, J.: Traffic Accident Statistics (2019).

[2] NHTSA: U.S. DOT Advances Deployment Of Connected Vehicle Technology To Prevent Hundreds Of Thousands Of Crashes (2016).

[3] Sengupta, R., Rezaei, S., Shladover, S.E., Cody, D., Dickey, S. and Krishnan, H.: Cooperative Collision Warning Systems: Concept Definition and Experimental Implementation, Journal of Intelligent Transportation Systems, Vol.11, No.3, pp.143-155 (2007).

[4] Verroios, V., Vicente, C.R. and Delis, A.: Alerting for Vehicles Demonstrating Hazardous Driving Behavior, Proc. 11th ACM International Workshop on Data Engineering for Wireless and Mobile Access, MobiDE'12, pp.15-22, ACM (2012).

[5] Böhm, A., Jonsson, M. and Uhlemann, E.: Co-existing Periodic Beaconing and Hazard Warnings in IEEE 802.11P-based Platooning Applications, Proc. 10th ACM International Workshop on Vehicular Inter-networking, Systems, and Applications, VANET'13, pp.99-102, ACM (2013).

[6] Yang, X., Liu, J., Vaidya, N. and Zhao, F.: A vehicle-to-vehicle communication protocol for cooperative collision warning, Ist Annual International Conference on Mobile and Ubiquitous Systems: Networking and Services 2004, MOBIQUITOUS 2004, pp.114-123 (2004).

[7] Rakotonirainy, A., Schroeter, R. and Soro, A.: Three social car visions to improve driver behaviour, Pervasive and Mobile Computing, Vol.14, pp.147-160 (2014).

[8] Renner, L. and Johansson, B.: Driver Coordination in Complex Traffic Environments, Proc. 13th Eurpoean Conference on Cognitive Ergonomics: Trust and Control in Complex Socio-technical Systems, ECCE '06, pp.35-40, ACM (2006).

[9] Currano, R., Park, S.Y., Domingo, L., Garcia-Mancilla, J., SantanaMancilla, P.C., Gonzalez, V.M. and Ju, W.: ¡Vamos!: Observations of Pedestrian Interactions with Driverless Cars in Mexico, Proc. 10th International Conference on Automotive User Interfaces and Interactive Vehicular Applications, AutomotiveUI' 18, pp.210-220, ACM (2018).

[10] Li, Y., Dikmen, M., Hussein, T.G., Wang, Y. and Burns, C.: To Cross or Not to Cross: Urgency-Based External Warning Displays on Autonomous Vehicles to Improve Pedestrian Crossing Safety, Proc. 10th International Conference on Automotive User Interfaces and Interactive Vehicular Applications, AutomotiveUI '18, pp.188-197, ACM (2018).

[11] Reig, S., Norman, S., Morales, C.G., Das, S., Steinfeld, A. and Forlizzi, J.: A Field Study of Pedestrians and Autonomous Vehicles, Proc. 10th International Conference on Automotive User Interfaces and Interactive Vehicular Applications, AutomotiveUI '18, pp.198209, ACM (2018).

[12] Lamas, R., Burnett, G., Cobb, S. and Harvey, C.: Driver Link-up: Exploring User Requirements for a Driver-to-Driver Communication Device, Proc. 6th International Conference on Automotive User Interfaces and Interactive Vehicular Applications, AutomotiveUI '14, pp.5:1-5:5, ACM (2014).

[13] Hagiya, T. and Nawa, K.: Acceptability Evaluation of Inter-Driver Interaction System via a Driving Agent Using Vehicle-to-Vehicle Communication, Proc. 11th Augmented Human International Conference, AH'20, ACM (2020).

[14] Ishibashi, M., Okuwa, M., Doi, S. and Akamatsu, M.: Indices for characterizing driving style and their relevance to car following behavior, SICE Annual Conference 2007, pp.1132-1137 (2007).

[15] Dey, D., Martens, M., Wang, C., Ros, F. and Terken, J.: Interface Concepts for Intent Communication from Autonomous Vehicles to Vulnerable Road Users, Adjunct Proc. 10th International Conference on Automotive User Interfaces and Interactive Vehicular Applications, AutomotiveUI'18, pp.82-86, ACM (2018).

[16] Harding, R., Morgan, F., Indermaur, D., Ferrante, A. and Blagg, H.: Road Rage and the Epidemiology of Violence: Something Old, Something New, Studies on Crime and Crime Prevention, Vol.7, No.2, pp.221-238 (1998).

[17] Makanae, K. and Sato, S.: Development of an Inter-driver Communication System Based on Inter-vehicle Communication Technology (2006).

[18] Yeong, D.J., Velasco-Hernandez, G., Barry, J. and Walsh, J.: Sensor and Sensor Fusion Technology in Autonomous Vehicles: A Review, Sensors, Vol.21, No.6 (2021).

[19] Wischhof, L., Ebner, A. and Rohling, H.: Information dissemination in self-organizing intervehicle networks, IEEE Trans. Intelligent Transportation Systems, Vol.6, No.1, pp.90-101 (online), DOI: 10.1109/TITS.2004.842407 (2005).
[20] Wang, C., Terken, J. and Hu, J.: CarNote: Reducing Misunderstanding Between Drivers by Digital Augmentation, Proc. 22nd International Conference on Intelligent User Interfaces, IUI '17, pp.85-94, ACM (2017).

[21] Wang, C., Terken, J., Hu, J. and Rauterberg, M.: "Likes" and "Dislikes" on the Road: A Social Feedback System for Improving Driving Behavior, Proc. 8th International Conference on Automotive User Interfaces and Interactive Vehicular Applications, AutomotiveUI '16, pp.43-50, ACM (2016).

[22] 5GAA: 5GAA Releases White Paper on C-V2X Use Cases: Methodology, Examples and Service Level Requirements.

[23] NHTSA: Vehicle-to-Vehicle Communications: Readiness of V2V Technology for Application (2014).

[24] Ho, K.Y., Kang, P.C., Hsu, C.H. and Lin, C.H.: Implementation of WAVE/DSRC Devices for vehicular communications, $2010 \mathrm{In}$ ternational Symposium on Computer, Communication, Control and Automation (3CA), Vol.2, pp.522-525 (online), DOI: 10.1109/3CA. 2010.5533694 (2010).

[25] Ministry of Land, Infrastructure, T. and Tourism: Guidelines for communication-based driving support systems (in Japanese) (2011).

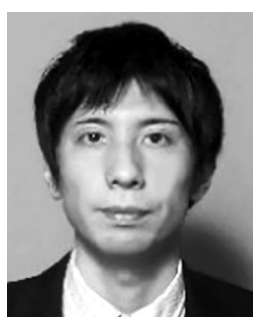

Toshiyuki Hagiya received B.E. in 2008, M.E. in 2010, and Ph.D. in 2018 degrees from Kyoto University. Since 2010, he has worked for KDDI Corporation and KDDI Research, Inc. From 2018 to 2021, he was seconded to Toyota Motor Corporation. He has been engaged in research and development of spoken dialogue systems, interactive user interface, and ITS. He received the IPSJ Outstanding Paper Award in 2018. He is a member of IPSJ, ACM.

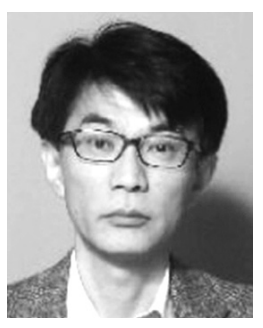

Kazunari Nawa received M.E. degree in polymer science from the University of Kyoto in 1987, and Ph.D. in material science from the University of Gunma in 1998. He joined R\&D center of Sumitomo Metal in 1987, where he was engaged in the research on electronic devices of LCD and Li-ion battery. From 2003, he joined Toyota InfoTechnology Center, where he was engaged in the technological area of human-vehicle interaction based on intelligent signal processing technologies. From 2017, he joined Toyota Motor Corporation and has been researching ITS technological area to obtain smooth traffic flow. Received best paper award of IEEE Cyber 2012 and 2016. 